\title{
Gene expression regulation: lessons from noncoding RNAs
}

\author{
RITA MIHAILESCU \\ Department of Chemistry and Biochemistry, Duquesne University, Pittsburgh, Pennsylvania 15282, USA
}

Our view of RNA function changed a great deal from the discovery of messenger RNA (mRNA), transfer RNA (tRNA), ribosomal RNA (rRNA), the deciphering of the genetic code, and of the roles these RNAs play in translation, during the 1950-1965 gold years. The 1960s-1980s period shone the spotlight on mRNA processing events and small RNAs that are part of small RNA-protein complexes essential for splicing. The discovery of RNA catalytic activity in the early 1980s transformed our thinking about RNA function, and this was just the beginning, as the discovery of both short and long noncoding RNAs in the 1990s and after 2000 triggered a new revolution in the RNA field.

The microRNA (miRNA) story started with the discoveries in the Ambros and Ruvkun labs in the late 1980s-early 1990s that in C. elegans, lin-4, a repressor of the protein encoding lin-14, did not contain a conventional open reading frame, hence not encoding for a normal regulatory protein, but rather for a small 22 nucleotide noncoding RNA partially complementary in sequence to a stretch in the $3^{\prime}$-UTR of lin-14. This mechanism of gene expression regulation by a small RNA was unprecedented, but its generality was not clearly inferred, as research in the next decade did not bring an abundance of evidence of similar pairs of small noncoding RNAs that regulate protein expression through complementarity with $3^{\prime}$-UTR sequences in their mRNA targets.

The discovery by Fire, Mello, and co-workers of RNA interference (RNAi), a process that involved small RNAs with a similar length to lin-4, hinted that this RNA too could have been generated by the same mechanisms operating in RNAi, and moreover, that other small noncoding RNAs that regulate gene expression might exist in C. elegans. This prediction was validated by the findings in the Ruvkun lab in 2000 of a second 21 nucleotide RNA, let-7, that controlled the expression of $l i n-41$ by partial complementarity with a stretch of nucleotide in its $3^{\prime}$ UTR. Unlike the small noncoding RNAs involved in RNAi, that Hamilton and Baulcombe discovered had perfect complementarity to their targets leading to target destruction, lin-4 and let-7 had only partial complementarity to their mRNA targets

Corresponding author: mihailescum@duq.edu

Article and publication date are at http://www.rnajournal.org/cgi/doi/ 10.1261/rna.050815.115. Freely available online through the RNA Open Access option.
3' UTRs, making difficult the search for their orthologs in other animals.

Nonetheless, the report by Ruvkun and co-workers in 2000 that let-7 RNA sequence is conserved across a wide range of animal phyla pointed to the universality of this mechanism of gene expression regulation by these small miRNAs and led to the quest for the discovery of other miRNAs in animals. The laboratories of Ambros, Bartel, and Tuschl reported in 2001 in back-to-back papers published in Science the identification of a new class of genes encoding for small noncoding RNAs with functions in gene expression regulation, leading to an explosion of the miRNA field.

As early as 2001 the involvement of the components of the RNAi pathways in the miRNAs biogenesis was demonstrated unequivocally. Work in the early 2000s also revealed that nucleotides 2-7 in the $5^{\prime}$-end of the miRNA, its "seed," are essential for mRNA target recognition, allowing for the prediction of miRNA target mRNAs by this complementarity. Furthermore, the accuracy of such predictions was greatly improved when it was discovered that sequences in the $3^{\prime}$ UTR of the miRNA are also important in mRNA target recognition. Since miRNA target recognition involves such short sequences it was recognized that an individual miRNA has the potential to regulate hundreds of mRNA targets, and these predictions were validated by experiments showing the deregulation of hundreds of genes as the result of the loss or overexpression of a miRNA in cell culture.

Although now it is clear that the function of the miRNArecruited RNA-induced silencing complex (RISC) is to repress protein synthesis off specific mRNA targets, by either mRNA destabilization or by translation inhibition, the mechanistic details of how this is achieved are still not fully elucidated. Genome wide studies of the influence of miRNAs on both, mRNA levels and translation efficiency, suggest that in fact which of these two mechanisms is the dominant path by which miRNA operates, depends on the system. Moreover, since the mid 2000s it is recognized that in coordination with the cell cycle, miRNAs can switch between translation repression and activation, as there are examples of miRNAs that target promoters of genes, inducing their expression.

(C) 2015 Mihailescu This article, published in $R N A$, is available under a Creative Commons License (Attribution-NonCommercial 4.0 International), as described at http://creativecommons.org/licenses/by-nc/4.0/. 
Our view of miRNA function also evolved, as now we acknowledge that they do not necessarily function as general on-off switches, but rather they are fine-tuning gene expression.

Perhaps in parallel with these studies trying to elucidate the mechanisms of action of miRNAs on gene expression, numerous other studies reported the link between miRNA expression dis-regulation and disease. The first study linking miRNA and cancer was published in 2002, and it is now known that in virtually almost every type of cancer there is altered expression of one or more miRNAs, that could function either as oncogenes or tumor suppressors. First reports implicating miRNAs in human cardiovascular diseases appeared in 2006, in autoimmune diseases in 2007, and in neurodegenerative diseases also in 2007 . The connections between miRNA expression dis-regulation and disease prompted researchers to address the mechanisms that regulate the expression levels of miRNAs themselves, and as early as 2006 it has been discovered that epigenetic mechanisms such as DNA methylation and histone modifications control the expression of some miRNAs.

The potential of miRNA-based therapeutic approaches has also been recognized, both miRNA-replacement and miRNA-targeting strategies being explored, currently miRNA mimics and miRNA antisense oligos being in different phases of clinical trials.

Although the miRNA field evolved tremendously since the early 1990s, researchers continue to be intrigued by the miRNA function in both normal and diseased systems, this area of the RNA field remaining of high interest and priority.

Since 2009, another class of regulatory RNAs, long noncoding RNAs (lncRNAs), has been discovered. The biological functions of lncRNAs are not well characterized, but emerging reports point toward their dual function as binders of both DNA/RNA and of proteins. Thus, they have been proposed to act as molecular scaffolds on which regulatory proteins can assemble, for example being implicated in regu- lating gene expression epigenetically through their interactions with chromatin modifier proteins. LncRNAs have also been shown to form RNA-protein complexes with transcription factors, mediating their activity. Additionally, lncRNAs have been implicated in the processing of mRNAs, regulating for example alternative splicing by forming RNA-RNA duplexes with the mRNA target that modulate the accessibility of cis-acting sequences important for splicing. Several studies also report the involvement of lncRNAs in translation regulation, by the formation of base pairs with the target mRNA, modulating its structure, but the molecular mechanisms of such regulation remain to be elucidated.

Interestingly, lncRNAs have been shown to regulate miRNAs function either by competing for binding to similar sequences within the target mRNA, or by binding directly protein partners that mediate the miRNA function.

In contrast to the multiple lines of evidence that link miRNAs with human disease, connections between lncRNAs and disease are just now emerging. The dis-regulation of lncRNAs expression has been linked to Alzheimer's disease and other neurodegenerative diseases, cancer, cardiovascular disease, and the elucidation of the causative mechanisms is a focus of current research in the RNA field.

While this commentary highlights only the noncoding miRNAs and lncRNAs, tremendous progress has also been made in the RNA field in the last 20 years in elucidating mechanisms of mRNA processing, translation regulation, in determining the structures of numerous complexes formed by RNA-binding proteins with their cognate RNAs. Of interest for my laboratory, the RNA G quadruplex structure has been characterized, being implicated in translation regulation, alternative splicing, polyadenylation, and more recently, in miRNA pathways. It is my expectation that the future will bring not only progress in elucidating the molecular mechanisms of individual regulation processes at the transcription and translation levels, but also of the crosstalk networks that connect such regulatory processes. 

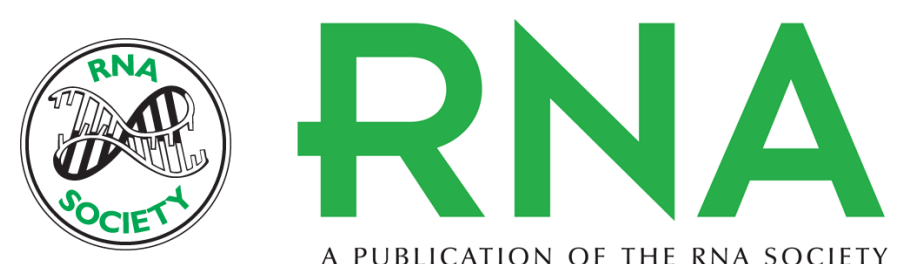

A PUBLICATION OF THE RNA SOCIETY

\section{Gene expression regulation: lessons from noncoding RNAs}

Rita Mihailescu

RNA 2015 21: 695-696

Open Access Freely available online through the RNA Open Access option.

Creative This article, published in $R N A$, is available under a Creative Commons License Commons (Attribution-NonCommercial 4.0 International), as described at License http://creativecommons.org/licenses/by-nc/4.0/.

Email Alerting Receive free email alerts when new articles cite this article - sign up in the box at the Service top right corner of the article or click here. 